\title{
SOME PROPERTIES OF THE CURVE STRAIGHTENING FLOW IN THE PLANE
}

\author{
ANDERS LINNÉR
}

\begin{abstract}
We will explicitly compute the gradient of the total squared curvature functional on a space of closed curves. An example shows that the flow along the gradient trajectory may cause curves to develop self-intersections. We prove the existence of strictly convex curves that momentarily turn nonconvex. In conclusion we use computer graphics to illustrate how self-intersections come about.
\end{abstract}

\section{INTRODUCTION}

Given a suitable space of closed curves in some Riemannian manifold, it is of nterest to study various functionals whose critical points include the geodesics. In particular the total squared curvature functional $F(\gamma)=\int_{\gamma} k^{2}$ and the length functional $L(\gamma)=\int_{\gamma}\left|\gamma^{\prime}\right|$ are examples of such functionals.

In recent years the flow along the negative gradient of $F$ has been labeled zurve straightening and along the negative " $L^{2}$ gradient" of $L$ curve shortening.

For $\gamma:[0,1] \rightarrow R^{2}$ let $v=\left|\gamma^{\prime}\right|=\left|\frac{d y}{d t}\right|$ and consider deformations $W=f N$ llong the unit normal $N$. We have

$$
\begin{aligned}
2 v \frac{\partial v}{\partial w} & =\frac{\partial v^{2}}{\partial w}=2\left\langle\frac{\partial^{2} \gamma}{\partial w \partial t}, \frac{\partial \gamma}{\partial t}\right\rangle_{R^{2}}=2\left\langle\frac{\partial(f N)}{\partial t}, \frac{\partial \gamma}{\partial t}\right\rangle_{R^{2}} \\
& =2\left\langle\frac{\partial f}{\partial t} N-f v k T, v T\right\rangle_{R^{2}}=-2 f v^{2} k .
\end{aligned}
$$

Here $T$ is the unit tangent vector and $k$ is the curvature. It follows that

$$
D L(\gamma) W=\frac{d L}{d w}=-\int_{0}^{1} f k v d t=-\int_{\gamma} f k=\langle W,-k N\rangle_{L^{2}} .
$$

This computation shows that the flow given by $\frac{\partial \gamma}{\partial w}=k N$ can be thought of as he negative " $L^{2}$ gradient" of $L$.

On the other hand the gradient of an arbitrary smooth functional $G$ on a Riemannian manifold $M$ is defined by the relation

$$
D G(p) W=\langle\nabla G(p), W\rangle_{p} .
$$

Received by the editors December 8, 1987.

1980 Mathematics Subject Classification (1985 Revision). Primary 58F25; Secondary 58E10, ;3C21.

Key words and phrases. Curve straightening, total squared curvature, gradient flow. 
Here $W \in T_{p} M$ and $\langle,\rangle_{p}$ is the inner product on $T_{p} M$. In this paper we will let the manifold be a space of closed curves in the plane with a specific Riemannian metric. We then compute $\nabla F$ using this metric.

The two flows, when considered on curves in the plane, will under very general assumptions carry initial curves to the circle. For instance

* Curve straightening will carry every immersion of the circle of rotation number one, into the circle in the infinite time limit [1].

* Curve shortening will make convex curves circular asymptotically [2]. Also simple closed curves with finitely many inflection points flow to the circle [3].

In $\S 1$ we will, (following [1] closely), define a Hilbert manifold $\Omega_{B}$ representing unit speed and unit length closed curves. The gradient of $\int_{\gamma} k^{2}$ on this space will then be computed explicitly, and the evolution equations follow immediately.

$\S 2$ will give an explicit example of an embedded curve that develops selfintersections under curve straightening. For curve shortening embedded curves stay embedded provided the curvature stays bounded [2].

In $\S 3$ we will show the existence of strictly convex curves that momentarily turn nonconvex under curve straightening. A surprise since symmetric convex curves remain convex [1]. Convex curves remain convex under curve shortening [4 and 2].

$\S 4$ contains computer graphics illustrating the result of $\S 2$.

The author wish to thank David A. Singer and Joel Langer for their relentless support and encouragement during the preparation of this paper. He also wish to thank Leo Schneider at John Carroll University for valuable technical assistance.

\section{Evolution EQUATIONS}

We will from now on restrict our attention to closed, unit speed, unit length curves $\gamma: I \rightarrow R^{2}$ where $I=[0,1]$. The centre of gravity will be assumed to be at the origin, i.e. $\int_{I} \gamma_{w}=0$. The subscript is to indicate the quantity at flow time $w$. No subscript means $w=0$.

A curve $\gamma$ will be represented by its indicatrix $\theta: I \rightarrow R$ so that $\gamma^{\prime}=\left(x^{\prime}, y^{\prime}\right)=(\cos \theta, \sin \theta)$. We use ${ }^{\prime}$ to denote derivative with respect to the arclength parameter throughout the paper. For $F$, the total squared curvature functional, we have

$$
F(\gamma)=\int_{\gamma^{\prime}} k^{2}=\int_{I} \theta^{\prime 2}=2 J(\theta)
$$

where $J$ is the energy functional $\left(k=\frac{d \theta}{d s}\right)$. We will deal with $J$ rather than $F$ when working with indicatrices.

We let

$$
H=\left\{\theta: I \rightarrow R \mid \theta(0)=0, \theta \text { absolutely continuous, } \theta^{\prime} \in L^{2}[0,1]\right\}
$$


with inner product $\langle\theta, \widetilde{\theta}\rangle_{H}=\left\langle\theta^{\prime}, \tilde{\theta}^{\prime}\right\rangle_{L^{2}}$ be our ambient Hilbert space on which $J$ makes sense. $L^{2}[0,1]$ denotes the set of real valued functions defined on $[0,1]$ whose square is Lebesgue integrable. The inner product of two such functions $f$ and $g$ is given by $\langle f, g\rangle_{L^{2}}=\int_{0}^{1} f(s) g(s) d s$. The indicatrices representing closed curves are in

$$
\Omega_{B}=\left\{\theta \in H \mid \theta(1)=\eta \cdot 2 \pi, \eta \in Z, \int_{I} \cos \theta=\int_{I} \sin \theta=0\right\} .
$$

It is a consequence of the implicit function theorem that $\Omega_{B}$ is submanifold of $H$. We note that indicatrices of different rotation number $\eta$ are in different components, and if $v \in T_{\theta} \Omega_{B}$ then $v$ is a function that vanishes at the end points and

$$
\langle v, \cos \theta\rangle_{L^{2}}=\langle v, \sin \theta\rangle_{L^{2}}=0 .
$$

The two-dimensional collection of functionals $\Lambda: H \rightarrow R$ given by

$$
\Lambda(\theta)=\int_{I} \lambda \cdot(\cos \theta, \sin \theta) \quad\left(\lambda \text { in } R^{2}, \quad \cdot=\operatorname{dot} \text { product in } R^{2}\right)
$$

has $\Omega_{B}$ as a zero level set. Letting $J_{B}=\left.J\right|_{\Omega_{B}}\left(J\right.$ restricted to $\left.\Omega_{B}\right)$ we can write

$$
\nabla J(\theta)=\nabla J_{B}(\theta)+\nabla \Lambda(\theta)
$$

for some $\lambda$.

Next we recall the duBois-Reymonds lemma: If $f$ is a continuous function and $\left\langle f, v^{\prime}\right\rangle_{L^{2}}=0$ for all $v$ vanishing at the end points and with continuous derivative then $f$ is constant. Indeed $v(s)=\int_{0}^{s}\left(f(t)-\int_{I} f\right) d t$ is one such $v$ and $v^{\prime}(s)=f(s)-\int_{I} f$ so

$$
0=\left\langle v^{\prime}+\int_{I} f, v^{\prime}\right\rangle_{L^{2}}=\left\langle v^{\prime}, v^{\prime}\right\rangle_{L^{2}} .
$$

This implies $v^{\prime}$ is zero so $f$ is constant.

Introducing $\alpha=\nabla J(\theta)$ and $\beta=\nabla \Lambda(\theta)$ we have, on the one hand,

$$
\begin{aligned}
& D J(\theta) v=\langle\alpha, v\rangle_{H}=\left\langle\alpha^{\prime}, v^{\prime}\right\rangle_{L^{2}}, \\
& D \Lambda(\theta) v=\langle\beta, v\rangle_{H}=\left\langle\beta^{\prime}, v^{\prime}\right\rangle_{L^{2}}
\end{aligned}
$$

by the definition of the gradient.

On the other hand, computing the derivatives we get

$$
\begin{aligned}
& D J(\theta) v=\left\langle\theta^{\prime}, v^{\prime}\right\rangle_{L^{2}}, \\
& D \Lambda(\theta) v=-\left\langle\lambda_{\perp} \cdot \gamma^{\prime}, v\right\rangle_{L^{2}}=\left\langle\lambda_{\perp} \cdot(\gamma-\gamma(0)), v^{\prime}\right\rangle_{L^{2}}
\end{aligned}
$$

(the last equality follows from integration by parts, $\perp$ indicates counter clockwise rotation by $\frac{\pi}{2}$ ).

Putting things together:

$$
\left\langle\alpha^{\prime}-\theta^{\prime}, v^{\prime}\right\rangle_{L^{2}}=0, \quad\left\langle\beta^{\prime}-\lambda_{\perp} \cdot(\gamma-\gamma(0)), v^{\prime}\right\rangle_{L^{2}}=0 .
$$


These equalities are true for all $v \in T_{\theta} H$ in particular for $v$ 's with continuous derivatives and vanishing at the end points. By the duBois-Reymonds lemma we may conclude that

$$
\alpha(s)=\theta(s)+c s, \quad \beta(s)=\lambda_{\perp} \cdot \int_{0}^{s}(\gamma(t)-\gamma(0)) d t+\tilde{c} s
$$

for constants $c$ and $\tilde{c}$. Also note that for $\theta$ 's in $\Omega_{B}$ the end points are fixed so $\alpha(1)=\beta(1)=0$, and we arrive at

$$
\alpha(s)=\theta(s)-\eta \cdot 2 \pi s, \quad \beta(s)=\lambda_{\perp} \cdot \int_{0}^{s} \gamma(t) d t .
$$

Now $\lambda$ has to be chosen so that $\alpha-\beta=\nabla J_{B}(\theta) \in T_{\theta} \Omega_{B}$. By (1) this implies $\int_{I} \alpha \gamma^{\prime}=\int_{I} \beta \gamma^{\prime}$ or after integration by parts

$$
\int_{I} k \gamma=\mathbf{I}\left(\lambda_{\perp}\right) \text {. }
$$

Here we have introduced the inertia tensor, $\left(\operatorname{det}(\mathbf{I}) \cdot \mathbf{I}^{-1}\right.$ is known as the inertia tensor [5])

$$
\mathbf{I}=\left(\begin{array}{ll}
\int_{I} x^{2} & \int_{I} x y \\
\int_{I} x y & \int_{I} y^{2}
\end{array}\right)
$$

which describes the mass distribution of $\gamma$.

Since by Cauchy-Schwarz inequality $\left(\int_{I} x y\right)^{2} \leq \int_{I} x^{2} \cdot \int_{I} y^{2}$ with equality if and only if $y$ is a scalar multiple of $x$ we see that $\mathbf{I}$ is invertible for closed curves. We have derived

Proposition (1.1). For $\theta \in \Omega_{B}$ with $\eta \cdot 2 \pi=\theta(1)$

(i) $\nabla J(\theta) \in T_{\theta} H$ is given by $\nabla J(\theta)(s)=\theta(s)-\eta \cdot 2 \pi s$,

(ii) $\nabla \Lambda(\theta) \in T_{\theta} H$ is given by $\nabla \Lambda(\theta)(s)=\lambda_{\perp} \cdot \int_{0}^{s} \gamma(t) d t$,

(iii) $\nabla J_{B}(\theta) \in T_{\theta} \Omega_{B}$ is given by

$$
\nabla J_{B}(\theta)(s)=\theta(s)-\eta \cdot 2 \pi s-\mathbf{I}^{-1}\left(\int_{I} k \gamma\right) \cdot \int_{0}^{s} \gamma(t) d t .
$$

Corollary (1.2). The flow equations associated with $-\nabla J_{B}$ are

$$
\begin{aligned}
& \frac{\partial \theta_{w}}{\partial w}(s)=-\theta_{w}(s)+\eta \cdot 2 \pi s+\mathbf{I}_{w}^{-1}\left(\int_{I} k_{w} \gamma_{w}\right) \cdot \int_{0}^{s} \gamma_{w}(t) d t, \\
& \frac{\partial k_{w}}{\partial w}(s)=-k_{w}(s)+\eta \cdot 2 \pi+\mathbf{I}_{w}^{-1}\left(\int_{I} k_{w} \gamma_{w}\right) \cdot \gamma_{w}(s) .
\end{aligned}
$$

Proof of (1.2). (i) is immediate, (ii) follows from

$$
\frac{\partial}{\partial s}\left(\frac{\partial \theta}{\partial w}\right)=\frac{\partial}{\partial w}\left(\frac{\partial \theta}{\partial s}\right) \text {. }
$$

Remark. If we only assume $\int_{I} \gamma_{w}=0$ when $w=0$ and instead assume $\gamma_{w}(0)$ fixed for all $w$ (i.e. fixed initial point), then since

$$
\frac{\partial}{\partial w}\left(\frac{\partial \gamma_{w}}{\partial s}\right)=\frac{\partial}{\partial s}\left(\frac{\partial \gamma_{w}}{\partial w}\right)
$$


we get

$$
\frac{\partial \gamma_{w}}{\partial w}(s)=\left(\left(\eta \cdot 2 \pi s-\theta_{w}(s)\right) \gamma_{w}(s)+\int_{0}^{s}\left(k_{w}(t)-\eta \cdot 2 \pi\right) \gamma_{w}(t) d t\right)_{\perp}
$$

under $-\nabla J(\theta)$. In particular

$$
\frac{\partial \gamma}{\partial w}(1)=\left(\int_{I} k \gamma\right)_{\perp}
$$

which offers some intuition for the quantity $\int_{I} k \gamma$.

Under $\nabla \Lambda(\theta)$ we get

$$
\frac{\partial \gamma_{w}}{\partial w}(s)=\left(\left(\lambda_{\perp} \cdot \int_{0}^{s} \gamma_{w}(t) d t\right) \gamma_{w}(s)-\int_{0}^{s}\left(\lambda_{\perp} \cdot \gamma_{w}(t)\right) \gamma_{w}(t) d t\right)_{\perp}
$$

and

$$
\frac{\partial \gamma}{\partial w}(1)=\left(-\int_{I}\left(\lambda_{\perp} \cdot \gamma\right) \gamma\right)_{\perp} .
$$

Since we want the curve to remain closed under $-\nabla J_{B}(\theta)$ we must have

$$
\frac{\partial \gamma_{w}}{\partial w}(0)=\frac{\partial \gamma_{w}}{\partial w}(1)
$$

so for $w=0$ we recover (2)

$$
\int_{I} k \gamma=\int_{I}\left(\lambda_{\perp} \cdot \gamma\right) \gamma=\mathbf{I}\left(\lambda_{\perp}\right)
$$

as expected.

\section{EMBEDDEDNESS}

This section will be devoted to the following

Counterexample (2.1). The curve with indicatrix

$$
\theta(s)= \begin{cases}0, & 0 \leq s<6 r=s_{1}, \\ -\frac{1}{5 r}\left(s-s_{1}\right), & s_{1} \leq s<s_{1}+5 \pi r=s_{2}, \\ -\frac{1}{3 r}\left(s-s_{2}\right)-\pi, & s_{2} \leq s<s_{2}+3 \pi r=s_{3}, \\ -\frac{1}{r}\left(s-s_{3}\right)-2 \pi, & s_{3} \leq s<s_{3}+\pi r=s_{4}, \\ \frac{1}{r}\left(s-s_{4}\right)-3 \pi, & s_{4} \leq s<s_{4}+\pi r=s_{5}, \\ \frac{1}{3 r}\left(s-s_{5}\right)-2 \pi, & s_{5} \leq s<s_{5}+3 \pi r=s_{6}, \\ \frac{1}{5 r}\left(s-s_{6}\right)-\pi, & s_{6} \leq s<s_{6}+5 \pi r=s_{7}, \\ \frac{1}{7 r}\left(s-s_{7}\right), & s_{7} \leq s<s_{7}+\frac{7 \pi r}{2}=s_{8}, \\ \frac{\pi}{2}, & s_{8} \leq s \leq \frac{1}{4}, \\ \pi-\theta\left(\frac{1}{2}-s\right), & \frac{1}{4}<s \leq \frac{1}{2}, \\ \pi+\theta\left(s-\frac{1}{2}\right), & \frac{1}{2}<s \leq 1,\end{cases}
$$




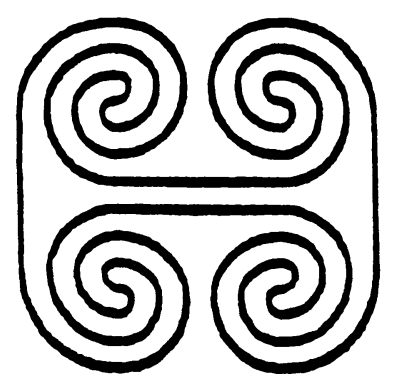

FIGURE 2.2

with $r=\frac{1}{2(24+43 \pi)}$ will represent a unit speed, unit length closed embedded curve that develops self-intersections under curve straightening. See Figure 2.2.

Lemma (2.3). Given an arbitrary indicatrix $\theta$, the proper choice of initial point in order to ensure centre of gravity at the origin is:

$$
\gamma(0)=\int_{0}^{1} s\left(\begin{array}{c}
\cos \theta(s) \\
\sin \theta(s)
\end{array}\right) d s .
$$

If $\gamma$ has $\pi$-rotational symmetry with $\theta(s)=\pi+\theta\left(s-\frac{1}{2}\right)\left(\frac{1}{2}<s \leq 1\right)$ then

$$
\gamma(0)=-\frac{1}{2} \int_{0}^{\frac{1}{2}}\left(\begin{array}{c}
\cos \theta(s) \\
\sin \theta(s)
\end{array}\right) d s .
$$

If in addition $\gamma$ is symmetric with respect to the $x$-axis with $\theta(s)=\pi-\theta\left(\frac{1}{2}-s\right)\left(\frac{1}{4}<s \leq \frac{1}{2}\right)$ then

$$
\gamma(0)=\left(\begin{array}{c}
0 \\
-\int_{0}^{\frac{1}{4}} \sin \theta(s) d s
\end{array}\right) .
$$

With the assumptions for (ii)

$$
\gamma\left(\frac{1}{2}\right)=-\gamma(0)
$$

Proof of (2.3). We only prove (i) explicitly. The proofs of (ii)-(iv) all involve change of variables and use of symmetry properties of sin and cos.

$$
\begin{aligned}
0 & =\int_{0}^{1} \gamma(s) d s=[s \gamma(s)]_{0}^{1}-\int_{0}^{1} s d \gamma \\
& =\gamma(1)-\int_{0}^{1} s \gamma^{\prime}(s) d s=\gamma(0)-\int_{0}^{1} s\left(\begin{array}{c}
\cos \theta(s) \\
\sin \theta(s)
\end{array}\right) d s .
\end{aligned}
$$

Lemma (2.4). If the rotation number $\eta=1$ and $\gamma$ has $\pi$-rotational symmetry with $\theta(s)=\pi+\theta\left(s-\frac{1}{2}\right), \frac{1}{2}<s \leq 1$, then the solution to the evolution equation (1.2(i)) is given by

$$
\theta_{w}(s)=(\theta(s)-2 \pi s) e^{-w}+2 \pi s .
$$

Also

$$
\theta_{w}(s)=\pi+\theta_{w}\left(s-\frac{1}{2}\right) \quad\left(\frac{1}{2}<s \leq 1\right) .
$$


And if $\gamma$ in addition is symmetric with respect to the $x$-axis with $\theta(s)=\pi-\theta\left(\frac{1}{2}-s\right)\left(\frac{1}{4}<s \leq \frac{1}{2}\right)$ then

$$
\theta_{w}(s)=\pi-\theta_{w}\left(\frac{1}{2}-s\right) \text {. }
$$

Proof of (2.4). We begin by defining two subsets of $\Omega_{B}$ corresponding to curves with $\pi$-rotational symmetry and also reflectional symmetry with respect to the $x$-axis. Let $S_{\pi} \subset \Omega_{B}$ be the set of indicatrices with the property $\theta(s)=\pi+$ $\theta\left(s-\frac{1}{2}\right) \quad\left(\frac{1}{2}<s \leq 1\right)$ and let $S_{r} \subset S_{\pi}$ be the set of indicatrices also satisfying $\theta(s)=\pi-\theta\left(\frac{1}{2}-s\right) \quad\left(\frac{1}{4}<s \leq \frac{1}{2}\right)$. We like to show that $S_{\pi}$ and $S_{r}$ are submanifolds of $\Omega_{B}$ and that $\nabla J_{B}(\theta) \in T_{\theta} S_{\pi}$ if $\theta \in S_{\pi}$ and $\nabla J_{B}(\theta) \in T_{\theta} S_{r}$ if $\theta \in S_{r}$.

From this (ii) and (iii) follow immediately and (i) follows since

$$
\begin{aligned}
\int_{0}^{1} k(s) \gamma(s) d s & =\int_{0}^{1} \gamma(s) d \theta(s) \\
& =2 \pi \gamma(1)-\int_{0}^{1} \theta(s) \gamma^{\prime}(s) d s \\
& =\int_{0}^{1}(2 \pi s-\theta(s)) \gamma^{\prime}(s) d s, \\
\int_{\frac{1}{2}}^{1}(2 \pi s-\theta(s)) \gamma^{\prime}(s) d s & =\int_{0}^{\frac{1}{2}}\left(2 \pi\left(s+\frac{1}{2}\right)-\theta\left(s+\frac{1}{2}\right)\right) \gamma^{\prime}\left(s+\frac{1}{2}\right) d s \\
& =-\int_{0}^{\frac{1}{2}}(2 \pi s+\pi-(\pi+\theta(s))) \gamma^{\prime}(s) d s \\
& =-\int_{0}^{\frac{1}{2}}(2 \pi s-\theta(s)) \gamma^{\prime}(s) d s
\end{aligned}
$$

implies that the term $\mathrm{I}^{-1}\left(\int_{I} k \gamma\right)$ is zero in the evolution equation (1.2(i)). This together with the fact that the needed symmetries are preserved, proves that

$$
\frac{\partial \theta_{w}}{\partial w}(s)=-\theta_{w}(s)+2 \pi s
$$

To prove that $S_{\pi}$ and $S_{r}$ are submanifolds we borrow the following argument from Palais [6]. Since $\Omega_{B}$ is a Riemannian manifold there is an exponential map exp: $V \rightarrow U$ mapping a neighborhood $V$ of zero in $T_{\theta} \Omega_{B}$ diffeomorphically onto a neighborhood $U$ of $\theta$ in $\Omega_{B}$ (geodesic normal coordinates). An isometry will preserve the connection, therefore geodesics are taken to geodesics. It follows that if $\phi: \Omega_{B} \rightarrow \Omega_{B}$ is such an isometry then $\phi \circ \exp =\exp \circ d \phi$. Now let $G$ be a group of such isometries and let

$$
\begin{gathered}
S=\left\{\theta \in \Omega_{B} \mid \phi(\theta)=\theta \text { for all } \phi \in G\right\}, \\
\widetilde{S}=\left\{v \in T_{\theta} \Omega_{B} \mid D \phi(\theta) v=v \text { for all } \phi \in G\right\} .
\end{gathered}
$$

We claim that $S \cap U=\exp (\tilde{S} \cap V)$. Since $\widetilde{S}$ is a linear subspace and $\theta$ is arbitrary it follows that $S$ is a submanifold. To prove the claim we let $\hat{\theta}$ be 
in $S \cap U$ and let $\exp (v)=\widehat{\theta}$ define $v$. Since $\exp (d \phi(\widehat{\theta}) v)=(\exp \circ d \phi(\widehat{\theta})) v=$ $(\phi \circ \exp ) v=\phi(\widehat{\theta})=\hat{\theta}=\exp (v)$ implies that $d \phi(\widehat{\theta}) v=v$ for all $\phi \in G$, the claim follows. Next we define two maps

$$
\begin{gathered}
\phi(\theta)(s)= \begin{cases}\theta\left(s+\frac{1}{2}\right)-\pi & 0 \leq s \leq \frac{1}{2}, \\
\theta\left(s-\frac{1}{2}\right)+\pi & \frac{1}{2}<s \leq 1,\end{cases} \\
\psi(\theta)(s)= \begin{cases}\pi-\theta\left(\frac{1}{2}-s\right) & 0 \leq s \leq \frac{1}{2}, \\
3 \pi-\theta\left(\frac{3}{2}-s\right) & \frac{1}{2}<s \leq 1 .\end{cases}
\end{gathered}
$$

Both are isometries of $\Omega_{B}$ and we have the two groups $G_{\pi}=\{\mathrm{id}, \phi\} \cong Z_{2}$ and $G_{r}=\{$ id $, \phi, \psi, \phi \psi\} \cong Z_{2} \oplus Z_{2}$ with corresponding $S$-sets $S_{\pi}$ and $S_{r}$.

Now suppose $v \in T_{\theta} \Omega_{B}$ and in the first case $v(s)=v\left(s-\frac{1}{2}\right)$ when $s \in\left(\frac{1}{2}, 1\right]$ and in the second case in addition $v(s)=-v\left(\frac{1}{2}-s\right)$ when $s \in\left(\frac{1}{4}, \frac{1}{2}\right]$. The equation $\theta_{w}(s)=w v(s)+\theta(s)$ defines a variation in $S_{\pi}$ if $\theta$ is in $S_{\pi}$ and in $S_{r}$ if $\theta$ is in $S_{r}$. Also $\partial \theta_{w} / \partial w=v$, so $v \in T_{\theta} S_{\pi}$ in the first case and $v \in T_{\theta} S_{r}$ in the second case. We finally note that $\nabla J_{B}(\theta)(s)=\theta(s)-2 \pi s$ in both cases which satisfies the relations for $v$ given above in the respective cases.

Proof of (2.1). By examining Figure 2.2 we see that the curve is made up of four similar pieces, each containing two straight line segments, six semicircles (of radii $r, 3 r, 5 r$ ) and a quarter of a circle (of radius $7 r$ ). It is clear the curve is embedded. By Lemma 2.3(iii) the initial point is on the $y$-axis and by Lemma 2.4 it will remain on the $y$-axis under the flow. By Lemma 2.3(iv) and Lemma $2.4 \gamma_{w}\left(\frac{1}{2}\right)=-\gamma_{w}(0)$ so it suffices to show that for some positive flow time $w, \gamma(0)$ is positive. This because initially $\gamma_{w}(0)$ is negative and by the continuity of the flow there must exist a $w$ such that $\gamma_{w}\left(\frac{1}{2}\right)=\gamma_{w}(0)$.

To find such $w$ we will use Lemma 2.3(iii) together with Lemma 2.4(i) and integrate explicitly. We will express the initial point in terms of nine integrals, with the relevant data:

$$
\begin{aligned}
& r=\frac{1}{2(24+43 \pi)}, \quad a_{i}=2 \pi\left(1+\alpha_{i} e^{-w}\right), \\
& I_{i}(w)=\frac{1}{\left(1+a_{i}\right)}\left[\cos \left(a_{i} t_{i+1}+\beta_{i} e^{-w}\right)-\cos \left(a_{i} t_{i}+\beta_{i} e^{-w}\right)\right], \\
& \alpha_{i}=\frac{k_{i}}{2 \pi}-1, \quad \beta_{i}=\theta_{i}-k_{i} t_{i}, \\
& y_{w}(0)=\sum_{i=0}^{8} I_{i}(w), \quad t_{9}=\frac{1}{4}, \\
& k_{0}=t_{0}=\theta_{0}=0 . \\
& 1
\end{aligned}
$$

A tedious evaluation shows that $y_{1}(0)>0\left(y_{1}(0) \approx 0.029\right)$. 


\section{CONVEXITY}

In this section we will establish

Counterexamples (3.1). There exist strictly convex curves that momentarily turn nonconvex under curve straightening.

First we need a few results on iscoceles triangular curves.

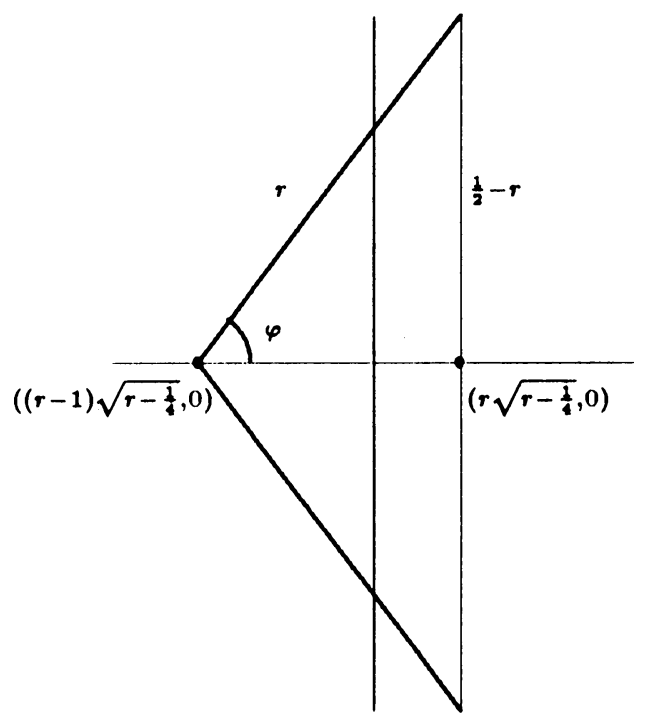

FIGURE 3.3

Lemma (3.2). The one-parameter family of isoceles triangular curves

$\gamma_{r}(s)= \begin{cases}\left(r R, s+r-\frac{1}{2}\right), & 0 \leq s \leq 1-2 r, \\ (s-(1-2 r))\left(\left(1-\frac{1}{r}\right) R, 0\right) & \\ \quad+(1-r-s)\left(R, \frac{1}{2 r}-1\right), & 1-2 r<s \leq 1-r, \\ (s-(1-r))\left(R, 1-\frac{1}{2 r}\right)+(1-s)\left(\left(1-\frac{1}{r}\right) R, 0\right), & 1-r<s \leq 1,\end{cases}$

with $R=\sqrt{r-\frac{1}{4}}$ and $r \in\left(\frac{1}{4}, \frac{1}{2}\right)$, (see Figure 3.3), have unit length and centre of gravity at the origin.

Proof of (3.2). $\gamma_{r}$ parametrizes the isoceles triangular curves with corners at $\left(r R, r-\frac{1}{2}\right),\left(r R, \frac{1}{2}-r\right)$ and $((r-1) R, 0)$ (see Figure 3.3) so each curve has unit length. Also, the centre of gravity of a line segment is at the mid point and therefore the centre of gravity of the triangles is

$$
2 r \cdot \frac{(r-1) R+r R}{2}+(1-2 r) r R=0
$$

in $x$ and by symmetry 0 in $y$.

Remark. The quantity $\int_{I} k \gamma=\int_{I} \gamma d \theta,\left(\frac{1}{2 \pi} \int_{I} \gamma d \theta\right.$ is known as Steiner's curvature centroid [7]), still makes sense for polygonal curves, the latter integral 
in Stieltjes sense. Also note that since $\gamma_{r}$ is symmetric around the $x$-axis $\int_{I} y d \theta=\int_{I} x y=0$ so the term

$$
\mathbf{I}^{-1}\left(\int_{I} k \gamma\right)=\mathbf{I}^{-1}\left(\int_{I} \gamma d \theta\right)
$$

simplifies to $\left(\int_{I} x d \theta / \int_{I} x^{2}, 0\right)$

Lemma (3.4). With $\gamma_{r}$ as in Lemma 3.2 and $R=\sqrt{r-\frac{1}{4}}$

$$
\begin{aligned}
& \text { (i) } \int_{I} x d \theta=2 R\left(\pi r-\arccos \left(\frac{1}{2 r}-1\right)\right), \\
& \text { (ii) } \int_{I} x^{2}=R^{2}\left(r^{2}(1-2 r)+\frac{2 r}{3}\left(3 r^{2}-3 r+1\right)\right) .
\end{aligned}
$$

Proof of (3.4). (i) With $\varphi$ as in Figure 3.3 we get, since $\theta$ is constant except at the corners,

$$
\int_{I} x d \theta=(\pi-2 \varphi)(r-1) R+2\left(\varphi+\frac{\pi}{2}\right) r R=2 R\left(\pi r-\arccos \left(\frac{1}{2 r}-1\right)\right) .
$$

Here we have used $r \sin \varphi=\frac{1}{2}-r$ and standard trigonometric identities.

(ii) Using the parametrization in Lemma 3.2

$$
\begin{aligned}
\int_{0}^{1-2 r} x^{2}(s) d s & =R^{2} r^{2}(1-2 r), \\
\int_{1-r}^{1} x^{2}(s) d s & =R^{2} \int_{1-r}^{1}\left(s-(1-r)+(1-s)\left(1-\frac{1}{r}\right)\right)^{2} d s \\
& =R^{2} \frac{r}{3}\left(3 r^{2}-3 r+1\right), \\
\int_{0}^{1} x^{2}(s) d s & =\int_{0}^{1-2 r} x^{2}(s) d s+2 \int_{1-r}^{1} x^{2}(s) d s .
\end{aligned}
$$

Remark. Since convex curves are characterized by having nonnegative signed curvature, and since the evolution equation is (Corollary 1.2(ii))

$$
\frac{\partial k_{w}}{\partial w}(s)=-k_{w}(s)+2 \pi+\mathbf{I}_{w}^{-1}\left(\int_{I} k_{w} \gamma_{w}\right) \cdot \gamma_{w}(s)
$$

we see that at points where $k_{w}(s)=0$ it is of interest to compare the last term with $-2 \pi$.

Lemma (3.5). For $\gamma_{r}$ as in Lemma 3.2 we have

$$
\lim _{r \rightarrow \frac{1^{+}}{4}}\left(\mathbf{I}^{-1}\left(\int_{I} \gamma_{r} d \theta\right) \cdot \gamma_{r}(1-r)\right)=\frac{9}{5}(-2 \pi) .
$$

Proof of (3.5). Using Lemmas 3.2 and 3.4 with $R=\sqrt{r-\frac{1}{4}}$ we get

$$
\begin{aligned}
\lim _{r \rightarrow \frac{1}{4}^{+}}\left(\mathbf{I}^{-1}\left(\int_{I} \gamma_{r} d \theta\right) \cdot \gamma_{r}(1-r)\right) & =\lim _{r \rightarrow \frac{1^{+}}{4}} \frac{2 R\left(\pi r-\arccos \left(\frac{1}{2 r}-1\right)\right)(r-1) R}{R^{2}\left(r^{2}(1-2 r)+\frac{2 r}{3}\left(3 r^{2}-3 r+1\right)\right)} \\
& =\frac{9}{5}(-2 \pi) .
\end{aligned}
$$



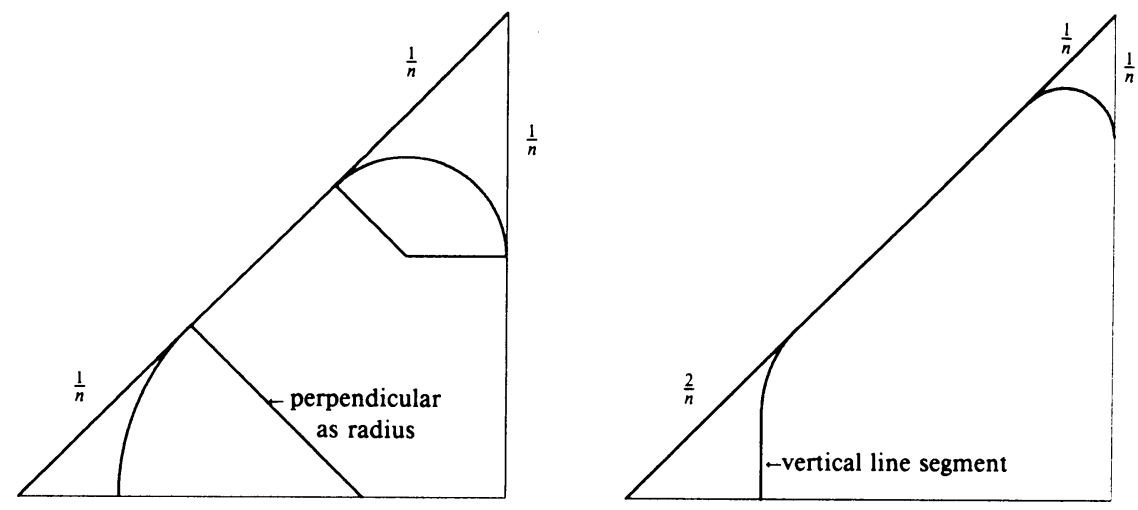

FIGURE 3.6

Proof of (3.1). The strategy of the proof is now clear. We will fix an $r_{0}$ such that

$$
\mathbf{I}^{-1}\left(\int_{I} \gamma_{r_{0}}\right) \cdot \gamma_{r_{0}}\left(1-r_{0}\right)=\frac{8}{5}(-2 \pi) .
$$

Next we will consider a sequence of convex curves $\gamma_{n}$, with corresponding indicatrices $\theta_{n} \in \Omega_{B}$, on each of which the curvature is bounded away from 0 except for a vertical line segment across the negative $x$-axis, with the property $\gamma_{n} \rightarrow \gamma_{r_{0}}$ pointwise. We will then show that for large $n$ 's

$$
\mathbf{I}^{-1}\left(\int_{I} k_{n} \gamma_{n}\right) \cdot \gamma_{n}\left(s_{n}\right)<\frac{7}{5}(-2 \pi) .
$$

Here $s_{n}$ is the parameter corresponding to the point where the line segment intersects the negative $x$-axis. Fixing one such $n=n_{0}$ we will have the curvature bounded away from zero except on the line segment. Also $\frac{\partial k}{\partial w}\left(s_{n_{0}}\right)<0$ for $\gamma_{n_{0}}$ at a point where the curvature is zero. It follows that if we follow the gradient trajectory in negative flow time we will instantly find a strictly convex curve whose trajectory will pass through $\gamma_{n}$ and then (at least momentarily) through nonconvex curves. Notice that the right-hand side of the evolution equation (Corollary 1.2(ii)) is constant for the parameters corresponding to the vertical line segment $\left(x_{n}(s)\right.$ is constant).

The only thing left to prove is the existence of such sequence $\gamma_{n}$ and that the relevant quantities converge to the corresponding quantities for $\gamma_{r_{0}}$.

The sequence we will consider will be built by circular arcs together with the vertical line segment across the negative $x$-axis. Starting from the triangular curve $\gamma_{r_{0}}$ we mark off the distance $\frac{1}{n}$ on each leg from the upper corner. Next we drop perpendiculars and note that the intersection will give the center for a circle tangent to the legs at the two marks. Do the same thing for the lower corner and for the corner on the $x$-axis we move the point of tangency a distance $\frac{2}{n}$ away from the corner which leaves room for the vertical line segment across the negative $x$-axis (see Figure 3.6). 
Note that for some of the parameters corresponding the three other line segments the curvature will increase rather than decrease. For instance the parameters corresponding to the vertical line segment across the positive $x$-axis will have this property. In this case following the gradient trajectory in negative flow time would not necessarily provide strictly convex curves. To avoid this problem we now describe how to replace these line segments with large circular arcs. We denote the length of the upper circular arc by $l_{n}$ we will cut off a piece of length $l_{n} / n$ at each end and again draw perpendiculars to find the center of the increasingly large circular arcs connecting the little circular arcs. The given sequence will converge pointwise to $\gamma_{r_{0}}$ and composing with the two homeomorphisms, scaling and translation, will produce a unit length sequence with centres of gravity at the origin which also converges pointwise to $\gamma_{r_{0}}$.

Next we turn our attention to the corresponding indicatrices $\theta_{n}$. They will converge pointwise to $\theta_{r_{0}}$ (and since they correspond to closed curves we know that $\left.\int_{I} \cos \theta_{n}=\int_{I} \sin \theta_{n}=0\right)$. Since

$$
\int_{0}^{1} k_{n}(s) x_{n}(s) d s=\int_{0}^{1}\left(2 \pi s-\theta_{n}(s)\right) \cos \theta_{n}(s) d s
$$

an application of the bounded convergence theorem will show convergence of this quantity, similarly for $\int_{0}^{1} x_{n}^{2}(s) d s$. Since the latter is not converging to zero the quotient of the two will converge to the corresponding quotient for $\gamma_{r_{0}}$. Finally we note that by rotating the curves $\frac{\pi}{2}$ the symmetry gives us $s_{n}=\frac{1}{2}$, and we have shown convergence of all the relevant quantities.

Remark. It should be pointed out that these curves are hard to visualize because they are extremely thin and the curvature is concentrated to very small portions of the curves.

\section{EXAMPLE}

We now give an example of (see Figure 4.1) the actual flow along the gradient trajectory of the curve in $\S 2$. Note that we used a different scale after the first eight curves. There is no problem implementing this since the symmetry allows for an explicit solution of the evolution equation. The flow time interval for the first eight pictures is 0.1 . There are other, not as symmetric, examples with the same characteristic spirals that also develop self-intersections under the flow.
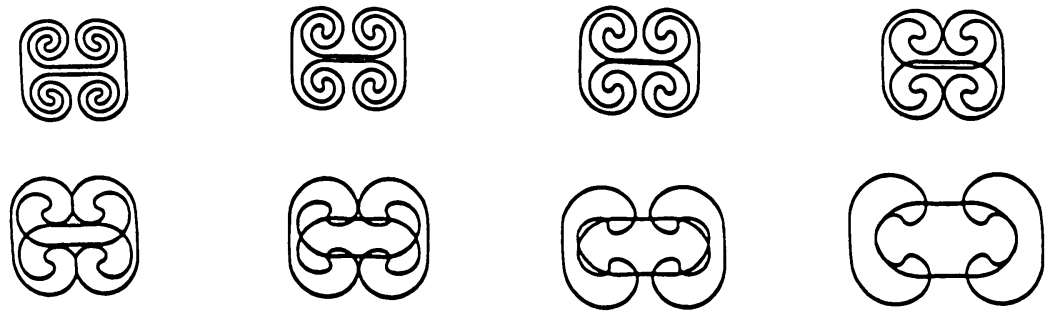

Figure 4.1 

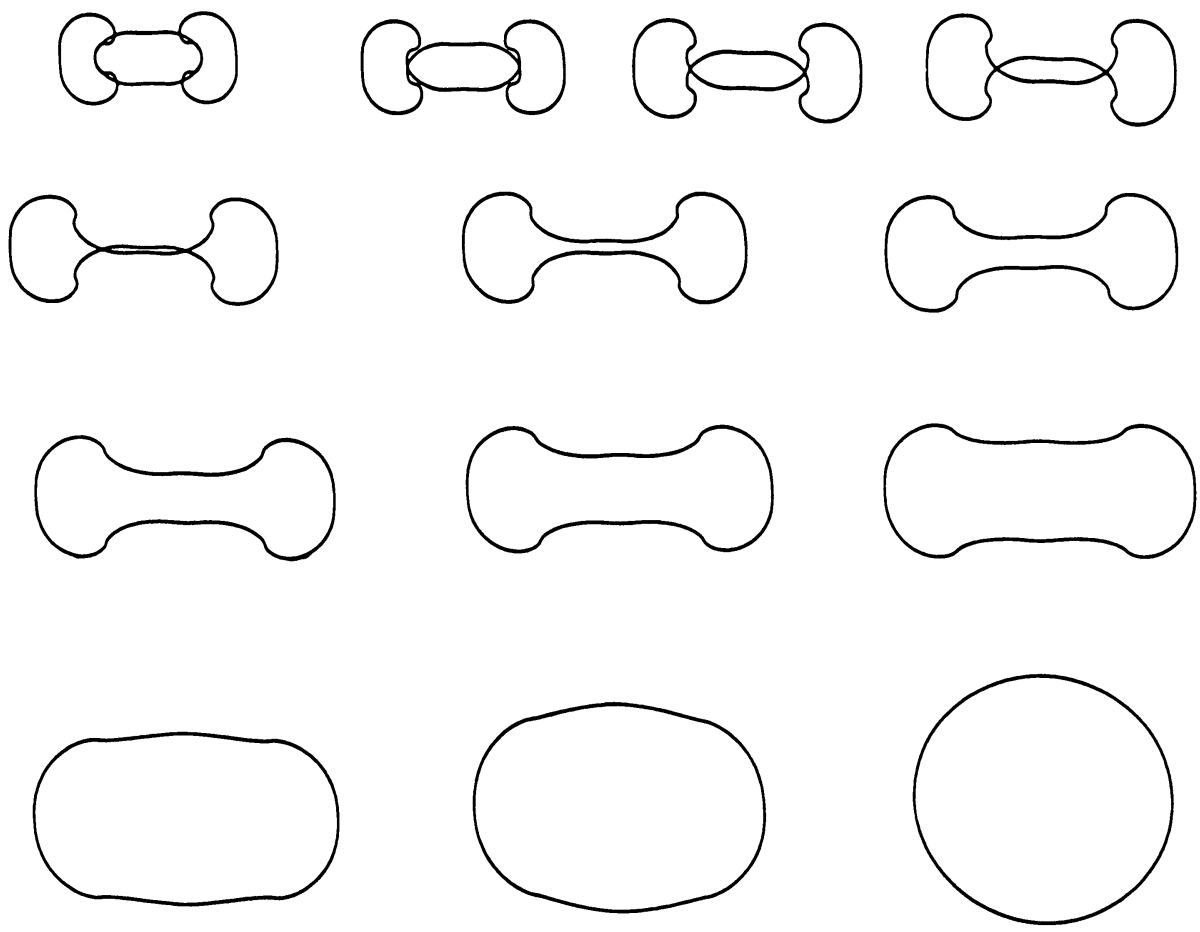

Figure 4.1 (CONTINUED)

\section{REFERENCES}

1. J. Langer and D. A. Singer, Curve straightening and a minimax argument for closed elastic curves, Topology 24 (1985), 75-88.

2. M. Gage and R. Hamilton, The heat equation shrinking convex plane curves, J. Differential Geometry 23 (1986), 69-96.

3. M. Grayson, The heat equation shrinks embedded plane curves to round points, J. Differential Geometry 26 (1987), 285-314.

4. M. Gage, Curve shortening makes convex curves circular, Invent. Math. 76 (1984), 357-364.

5. A. Linder, Föreläsningar $i$ statik, Institutionen för Mekanik, The Royal Institute of Technology, Stockholm, 1978,.

6. R. Palais, The principle of symmetric criticality, Comm. Math. Phys. 69 (1979), pp. 19-30.

7. S. Buchin, Lectures on differential geometry, World Scientific, Singapore, 1980, 19-30.

Department of Mathematics and Statistics, Case Western Reserve University, CleveLAND, OHIO 44106 